\title{
Las prácticas corporales y su relación con la pedagogía en la escuela colombiana en 1935.
}

\author{
Body practices and their relation to pedagogy in the colombian school system in 1935
}

\author{
Astrid Bibiana Rodriguez Cortés**
}

\begin{abstract}
Resumen
La Escuela en Colombia, hacia la década de 1930, acoge saberes científicos como la biología, la psicología experimental y la medicina. La renovación de la pedagogía se dio con la escuela nueva. De esta manera, los saberes científicos junto con esta nueva escuela buscarían configurar sujetos que sirvieran a la idea de progreso económico que tenía en mira la nación colombiana.

Las prácticas corporales, como la salud, la disciplina, la eugenesia, la urbanidad y las prácticas físicas, intervinieron el cuerpo de los estudiantes, y determinaron un sujeto escolar saludable, vigoroso, de carácter fuerte y respetuoso; circunscribieron el papel del maestro al de un centinela del cuidado del cuerpo, del bienestar de la salud y lucharon con la idea de regenerar una raza considerada
pobre, ignorante y enferma.
\end{abstract}

Palabras clave: Pedagogía, escuela nueva, prácticas corporales, cuerpo.

\section{Abstract}

During the thirties the Colombian school system took up scientific knowledges such as Biology, Experimental Psychology, and Medicine. Active school brought about a pedagogical renewal. Thus, scientific knowledge along with active school strived for shaping subjects that are suitable for the Nation's economic progress ideal.

Both body practices - such as health, discipline, eugenics, and urbanity- and physical practices played a role on the student's bodies, determining a healthy, vigorous, strong-charactered and respectful school subject. The teacher's role was confined to that of a guardian of body care, health and well-being, struggling against the idea to regenerate a poor, ignorant, ill race.

Key words: $\quad$ Pedagogy, active school, body practices, body.

Fecha de recepción: 16 de agosto de 2006

Fecha de aceptación: 20 de octubre de 2006

\footnotetext{
* Ponencia presentada en el congreso XIV Jornadas de Historia de la Educación, La Plata, Argentina, agosto de 2006, producto del proyecto de investigación financiado por el Centro de Investigaciones de la Universidad Pedagógica Nacional, CIUP, para la El lequipo en la Escuela de 1920 a 1950, en las Revistas de Educación en Colombia. El equipo de investigación está formado por los profesores Ximena Herrera, Víctor Jairo Chinchilla y Astrid Bibiana Rodríguez
y por las estudiantes Tania Robledo y Adriana Cortés.

** Profesora de la Facultad de Educación Física. Universidad Pedagógica Nacional. Magíster en Educación. astridbibianarc@gmail.com o astridbibianarc@hotmail.com
} 


\section{PONENCIAS}

La escuela en Colombia, hacia el decenio de 1930, con la llegada de la "llamada modernidad" (Saldarriaga, 1977: 22) pedagógica trae saberes como la biología, la psicología experimental, y la medicina, acogiendo la renovación de la pedagogía con la escuela nueva (llamada también escuela del juego y del trabajo). Esta escuela remplazaría el viejo sistema carcelario por un grupo social, orgánico, útil para la comunidad y el sujeto que en ella se perfeccionaba. El campo de sus enseñanzas no sería ya el aula fría y tétrica para el espíritu inmensamente sensitivo (Balcázar, 1935: 60-61), serían los campos, los talleres, las granjas quienes enseñarían al niño y a la niña el valor de estar en la escuela. De esta manera, los saberes científicos, junto con la escuela nueva, buscarían configurar sujetos que sirvieran a la idea de progreso económico que tenía en mira la nación colombiana.

Así las cosas, la educación se entendería como un problema esencialmente social, y las nuevas preocupaciones pedagógicas eran, sin duda, una consecuencia del anhelo de hacer de la vida algo que verdaderamente respondiera a un concepto humano, creando y afianzando la relación entre los seres humanos con base en el altruismo y la confraternidad, impregnando la acción educadora de un alto sentimiento de filantropía e igualdad (Op. cit: 58). Entre tanto, el papel de la pedagogía, al igual que el de otros saberes, era dar respuesta de manera científica y objetiva "al cómo y el porqué de las cosas, más que a la esencia de éstas" (Saldarriaga, 1997: 16). La pedagogía se regía por la psicología experimental, la cual proponía fundar laboratorios y traer misiones pedagógicas extranjeras con todos los métodos, procedimientos y sistemas de "mensura" intelectual.

La pedagogía debía partir del conocimiento anatomo-fisiológico del educando, con todo lo que esto significaba investigar en las hondas raíces de la herencia y el medio en que se desenvolvía el individuo. Para llevar a cabo este fin, el maestro necesitaba la colaboración del médico, o mejor, del biólogo porque éste era el único que podía penetrar hasta la constitución íntima del carácter, aproximarse más a la verdad en el problema de las aptitudes, de la formación de la personalidad, del funcionamiento del psiquismo y de sus perturbaciones, y de las más remotas influencias que pueden transmitirse de la evolución filogenética (Bernal Jiménez, 1935: 52-53).

Estos requerimientos de la sociedad por una escuela distinta hacían que la Facultad de Ciencias de la Educación deseara formar un tipo de profesor que reuniera la ciencia y la técnica en su transmisión; no pretendía formar diletantes sin profundidad en ningún campo del saber ni profesores eruditos en un microscópico sector de la ciencia. La especialización que perseguía la Facultad no era cercenamiento ni atomización de las facultades mentales; era concreción metódica del esfuerzo intelectual a un campo extenso de materias conexas, de acuerdo con las predilecciones especiales del alumno (Bernal Jiménez, 1935: 441).

Se sugería como método adecuado el del doctor Decroly, el cual centraba su trabajo en el interés del alumno, la enseñanza en un programa vasto y flexible que él llamaba de ideas asociadas, y que se desarrollaba por medio de centros de interés. Según Decroly, el programa del maestro debería interpretar las necesidades y la naturaleza del niño, y de acuerdo con éstas, él comprendería mejor su propia conservación y su propio desarrollo; el programa, el esfuerzo personal del alumno, e método y la buena preparación del maestro defendían la concepción de una nueva escuela (Pantoja, 1935: 346).

Así, entonces, la nueva escuela era un modelo a copiar en todo el país: una escuela sencilla, pero con una gran vitalidad, y que sería el motor de la pequeña colectividad donde se hallare. Allí, antes que aprender a leer, se le enseñaría al niño a vivir, en consonancia con las necesidades de su medio y en un ambiente donde sería tan grato el trabajo como el esparcimiento (Pantoja, 1935: 433). Lo más apremiante era preparar al nuevo tipo de maestro, el cual desde una orientación renovadora daría sentido social a la escuela, centrando su atención en el alumno, pensando en su bienestar y salud, lo que con propiedad ajustada a la realidad podría llamarse el punto neurálgico de la escuela pública. La salud infantil estaba siendo atacada por múltiples factores: por locales estrechos, sin luz y sin aire, por deficiente nutrición del organismo infantil, por carencia absoluta o efecto grave de higiene personal, por dolencias endémicas, por ignorancia o descuido en el hogar y de parte de las autoridades (Nieto, 1935: 420-421).

El papel del maestro residiría, entonces, en formar hábitos de defensa para el cuerpo y el espíritu, utilizando el libro como instrumento de cultura, el huerto y el taller como experiencia de trabajo y relación con el medio ambiente, la cooperación social como oportunidad de relación familiar, la educación corporal como espacio para fortalecer su cuerpo y disciplinar su mente. Todo ello propiciaría las oportunidades para cultivar la curiosidad en el niño con nuevos estímulos, los cuales ofrecerían situaciones que plantearían problemas para resolver y armonizar la educación moral, intelectual y física. De esta forma, ya el escolar no sería el simple 
memorizador de principios abstractos, sin otra finalidad próxima que la de recitarlos en los exámenes; sería el descubridor, hasta donde ello fuera posible, de conocimientos que le sirvieran como instrumentos de trabajo y de cultura (Ministerio de Educación Nacional, 1933: 303).

La escuela, centrada en estos preceptos, buscaría intervenir en la construcción del cuerpo de un sujeto social. Para ello abordaría diferentes prácticas corporales, como las prácticas de salud, las cuales centrarían su atención en la salud e higiene de los niños; las prácticas de disciplina, que buscarían que la educación reuniera sus enseñanzas en el autodominio; las prácticas de urbanidad, que pretenderían la buena conducta de los niños, las prácticas de eugenesia que procurarían mejorar la raza y el carácter; las prácticas intelectuales, que fortalecerían la inteligencia del individuo y, por último, las prácticas físicas, las cuales, en unión con las anteriores, formarían un ser integral.

\section{La salud infantil, responsabilidad del médico, del maestro y de la maestra}

El trabajo mancomunado del médico con el maestro fue indispensable, pues un maestro atento a las prácticas de salud podía distinguirse como el nuevo tipo de maestro que llevaría a la escuela la orientación renovada, con la misión del cuidado de la salud de los escolares y la estética de la escuela, entre otras. La salud del niño era una circunstancia angustiosa, por ello era preciso hacer de cada maestro un colaborador social, un centinela que diera la voz de alerta ante las entidades públicas y ante la sociedad, dondequiera que se presentara esta aflictiva situación, y contagiara de esta preocupación, en una acción sin reposo, a toda la colectividad.

La educación perseguía, entre otros objetivos, poner a los niños en íntimo contacto con su medio para que se capacitaran de la mejor manera y pudieran utilizarlo con saludables resultados. Era imprescindible que, por el sistema de los centros de interés, se propendiera a la modificación de ese medio, de suerte que llenara a satisfacción las necesidades de vida de la familia y de la comunidad en general. Había que despertar en los niños y las niñas hábitos de aseo, estética, gusto, etc., en relación con la escuela, la familia y el cuerpo (Jaime, 1935: 603); por ello, el maestro procuraría realizar con sus alumnos todos los juegos concernientes a la higiene personal, y después de la escuela se haría el balance para ver cómo se encontraba la salud y el cuerpo del niño.
Higiene, alimentación, calzado escolar, uso de uniformes escolares u overoles económicos, en lo que atañe a hechos puramente físicos; moral, buenos modales, conciencia ciudadana, en lo que toca a postulados del espíritu, son en síntesis ideales que lentamente podrían tener un feliz logro. Al niño debería enseñársele a sacar ventajas del conocimiento del alfabeto para su trabajo, para su progreso, paulatino, para su bienestar general. Esto implicaría una coordinación efectiva entre la instrucción pública y todas las demás realizaciones que en la comunidad social debía asumir el niño (N.D. S.F.: 93).

Con relación a la conciencia estética de la escuela, el maestro, debía tener la pulcritud y el buen gusto (dentro de la sencillez y aun dentro de la pobreza), la alegría del ambiente y, con todo esto, las normas sobrias de una pedagogía del buen sentido, pedagogía sin términos pedagógicos, vinculada a los intereses y realidades de la edad del niño y de las circunstancias de cada localidad (Nieto Caballero, 1935: 420-421). El buen aseo en los locales escolares, la ventilación, la luz, eran aspectos que se recordaban a los maestros para que tuvieran en cuenta en la organización de su institución.

A la educación, a la escuela y al maestro les correspondían el desarrollo de las fuerzas físicas, morales e intelectuales del pueblo colombiano. Enseñar a los estudiantes hábitos de limpieza y realizar campañas de higiene de uso profiláctico lograrían una defensa automática y natural frente a los peligros y a las enfermedades que les acechaban en su medio, así como "el dinamismo necesario para hacer de nuestra patria un pueblo grande" (Camargo Uribe, 1935: 615).

\section{La misión de la educación debe ser el autodominio}

El resultado de toda educación debía ser proporcionar al hombre una mayor felicidad, así como un mayor rendimiento individual, pero ello no podría conseguirse si no se lograba un autodominio y sentido de la responsabilidad:

la pereza mental y corporal que es entre nosotros germen de miseria espiritual y física, nace de la carencia de fuerza para dominarnos, y eso porque desde niños sólo aprendimos a hacer o pensar lo que se nos imponía como tarea cotidiana, pero no lo que uno mismo se impone y consigue con luchas interiores y fecundas (Castellanos, 1933: 107).

Los castigos corporales como medio de corrección y autodominio eran usados por padres y maestros: 


\section{PONENCIAS}

el azote, los puntapiés, las terribles amenazas, los adustos semblantes con que los padres, oprimien do aquella festiva alegría y viveza tan natural en los niños, como hijas de su inocencia, querían que fueran unos hipócritas y unos Catones circunspectos. ¿Y qué resulta por lo común de semejantes métodos? El menor mal será que se hagan embusteros, pues era evidente que el demasiado temor produciría siempre en el hombre estos efectos (Castellanos, 1993: 69).

La escuela ejercería en el niño:

la norma el rigor y el castigo, por ambiente la clausura y el silencio, la posición fatigosa y deformante en largas horas de labor, la quietud, la tristeza y el temor. Así el escuchar reglas y preceptos, todo un mero verbalismo convertían al estudiante en autómata que recita su lección, aislado de la vida que desde fuera tendría para él la más dulce sugestión (Balcázar, 1935: 60).

Los azotes, castigos, palmetas y ásperas amenazas que sólo producirían en los niños llanto y dolor, no eran parte de una enseñanza cristiana, ya que quién más para cultivar la dulzura y el amor en los niños sino Jesucristo; por ello, “QQuién creería que en el siglo XIX se aplicase la pena infamante del azote, impuesta por las leyes criminales a los malvados, a la corrección y castigo de unos niños todavía inocentes? (Sin autor, Discurso sobre la educación, N. D. S. F.: 68)

Por ello, la misión de la educación debería ser formar en los educandos su voluntad y energía, conduciendo todo ello al vencimiento de sí mismos, construyendo de esta manera el carácter de los futuros adultos. Esto no se lograría con absurdos sistemas de palo y rejo, el hombre debería aprender con la práctica y la experiencia, dones que poseía el buen maestro. Los sistemas de castigo se podrían comparar como lo que el irraciona aprende en el circo con un buen domador (Castellanos, 1933: 107)

Lograr enseñar el autodominio en los niños era ensenanza indispensable de un buen maestro, pero no con palo o rejo: era por medio de la experiencia, la voz dulce y los consejos que se lograría disciplinar el cuerpo y la mente. La nueva escuela llamaba la atención para mostrar que el castigo no era otra cosa que la represión de las emociones de los niños y, por ello, con métodos violentos no se cambiaría realmente su actitud, sino que se lograría hacer de ellos hipócritas que actuarían ante su familia y sus maestros.

\section{La conducta: el vivo reflejo de las enseñanzas escolares}

El niño debía observar fuera de la escuela una conducta que reflejara las enseñanzas escolares. Los más elementales actos de mala educación, la omisión del saludo, los juegos en las vías públicas, el vocabulario inadecuado o grosero, eran puntos vitales que los maestros y las juntas escolares debían propender por corregir y prestar para ello su valioso aporte. Se debían propugnar por las clases prácticas de urbanidad y buenos modales, las cuales debían permitir al ciudadano, no solamente enseñanzas puramente teóricas, sino brindar las herramientas para un comportamiento adecuado dentro y fuera de la escuela (Sin Autor, N. D. S. F.: 91)

Tanto la familia como la escuela debían ser la clave para formar niños y niñas de bien, que aprendieran a comportarse en sociedad; así:

Se impone la obligación de que los progenitores, por medio de conferencias con los encargados de la educación, se den cuenta de la manera como hoy se enseña y especialmente de los adelantos en la ciencia de corregir y de formar hábitos morales; de cómo el maestro necesitaba respeto, estímulo y cooperación de los hogares en la provisión de material, en la asistencia escolar y en los sistemas educativos (Balcázar, 1935: 67)

En el maestro se depositaba la formación especial que requería la mujer, pues de ella se esperaba "la niña dulce, inocente y tímida, flor oculta a quien un Don Juan descubre por la fragancia de sus virtudes" (Gómez Vega, 1935: 655). Éste era el tipo de mujer que los colegios femeninos formarían: mujer dulce, cándida y llena de virtudes, educadora del hogar, y del esposo y los hijos, que enseñaría todas aquellas buenas maneras que la sociedad requería.

La formación en hábitos, comportamiento y buena conducta dependían de la mancomunidad entre familia y escuela, así que era obligación del maestro formar a niños y padres para lograr en la sociedad los cambios de actitud. Un país en progreso necesitaba gente con buenos modales, con normas y reglas claras de proceder, con definiciones sobre la manera de actuar; por ello, la urbanidad penetró en todas las instituciones sociales y determinó un molde para el cuerpo del niño, una manera de asumirse mujer $u$ hombre, y una forma de pensar la sociedad colombiana. 


\section{El cuidado de la raza asegura la independencia económica de la nación}

La ansiedad por una raza enérgica que garantizara una nación fuerte se manifestaba en toda América Latina; por ello.

Don Enrique Molina, pedagogo, distinguidote de Chile, estudiante de los ideales de los tiempos presentes, sintetiza el objeto de la educación contemporánea en la siguiente forma: [La finalidad principal] de la educación es desarrollar la eficiencia social de los individuos. La eficiencia presenta dos grandes fases: la económica y la espiritual. La primera supone, por un lado, el cuidado del vigor físico de la raza y de su preparación técnica, para asegurar la independencia económica de la nación. La faz espiritual de la eficiencia que se persigue es la que debe dar al individuo una sólida estructura moral y cívica y cultivar su inteligencia y sus sentimientos en forma que sean para él fuentes de puros y serenos placeres e instrumentos de una noble actividad creadora (Arizala, 1935: 76).

La educación se convertía entonces en el bastión que permitiría salir de la ignorancia, la pobreza y la inferioridad racial a los pueblos; por ello se hacía necesario aumentar hasta donde fuera posible el número de las escuelas primarias, crear las nocturnas, fundar una casa de menores para los pequeños delincuentes, y desarrollar una segunda enseñanza que estuviera al alcance de las clases pobres: era preciso favorecer las escuelas-talleres, las granjas agrícolas y las bibliotecas infantiles; $y$, lo más urgente, era ineludible ir en socorro de la raza creando los servicios médicos y dentales en las escuelas, auxiliando generosamente todas las obras tendientes a combatir el hambre y las enfermedades entre los niños, y estableciendo las primeras colonias de vacaciones y los sanatorios infantiles (Gaviria, 1935: 143), así como incentivar en los jóvenes las prácticas de scout, las cuales profesaban ideales humanitarios y deseaban la paz entre los pueblos (Bernal Jiménez, 1935: 338).

En este sentido, el Gobierno emprendió proyectos como las reformas de la enseñanza primaria, secundaria, normalista y universitaria: "En nuestro concepto, la tan pregonada campaña de desanalfabetización -que más propiamente debe llamarse de alfabetización- constituye uno de los proyectos fundamentales de la difusión cultural" (Sin autor, N. D. S. F.: 67). Así como las campañas contra el alcoholismo o contra las enfermedades venéreas, hacían parte del fortalecimiento de la raza y las clementes virtudes.

\section{La educación moral, junto a la educación física e intelectual constituirían la completa formación del hombre}

La educación moral, junto con la física e intelectual, constituyen la completa formación del hombre, según los sabios principios de la pedagogía científica, pues con ella se persigue la cristalización de las buenas costumbres y también la formación de la voluntad y del carácter, factores esencialísimos de una recia personalidad humana (Cadavid Tobón, 1935: 199).

La preocupación por una educación integral llevó a discutir la forma en que se había venido abordando la educación, separando el cuerpo del espíritu, preocupándose más por la educación intelectual que por la educación del cuerpo.

La educación física debía robustecer el cuerpo y activar tanto las facultades sensoriales, como las sensitivas del niño, toda vez que las segundas están en relación directa con las primeras.

El principio muy sostenido y muy sostenible de Juvenal podría ser complementado diciendo: Voluntad firme y mente sana, en cuerpo sano. ¿Cuáles eran los objetivos para alcanzar este ideal de educación? Asegurar la actitud física del alumno, cultivar en la juventud sus facultades corporales y proporcionar al cuerpo la salud, mediante la práctica de reglas higiénicas, que le servirían de guía durante su transcurso por la vida para alcanzar así la armonía de todas las facultades (Cadavid Tobón, 1935: 197).

Entre tanto, la conservación de la salud se imponía como un deber esencial, pues aparte de que el niño la necesitaba, era de un gran valor social, comoquiera que la familia, la sociedad y la patria ningún provecho sacarían de un hombre débil y enfermizo, por más cultura y ciencia que tuviera.

Hombres fuertes, vigorosos, con voluntad firme, era la necesidad de la nación colombiana; debido a ello resultaba importante, luego del primer mes de asistencia de los estudiantes, que el maestro realizara testes (sic) de inteligencia y de desarrollo físico, para medir en sus alumnos la capacidad auditiva, visual, táctil, muscular, etc., de tal forma que se realizaran actividades que dieran mayor provecho al desarrollo mental y fisiológico de los estudiantes (Zapata, 1935: 170). Era necesario dar al maestro un año por lo menos, para que observara las actividades naturales de los niños como colectividad, 
y de cada niño en particular, lo cual requería que los alumnos concurrieran a los establecimientos de educación, y que, dentro de una disciplina metodizada, se los dejara ejecutar con libertad todos los actos de su vida ordinaria, sin que el maestro interviniera en enseñanza directa alguna, y sólo actuara como observador. Pasado el año de observación, se habría obtenido un resultado tan maravilloso que el maestro estaría en condiciones de asegurar que conocía el material con el cual iría a trabajar y los,medios con que habría de conseguir los altos fines educativos que justificarían los grandes gastos del Estado (Benavides, 1933: 228). La observación rigurosa se convertiría en la base del método pedagógico de los maestros de la nueva escuela, ya que ella les daría como resultado el conocimiento válido, encontrando las aptitudes y particularidades de los estudiantes.

La práctica de la clase de Educación Física tendría como fin el cuidado del cuerpo, ya que ella fortificaría los órganos favoreciendo un hombre sano, vigoroso y disciplinado; la práctica del ejercicio físico activaba el mayor flujo de la sangre a los órganos y músculos: esto favorecía la eliminación de las toxinas elaboradas en el organismo y regularizaba el mejor funcionamiento de los pulmones (Benavides, 1933: 197). La explicación fisiológica y científica del cuerpo era otro elemento que justificaría la presencia de la educación física escolar.

Mantener un cuerpo saludable y vigoroso permitiría también un espíritu fortalecido. "Montaigne decía: 'No es un alma ni un cuerpo lo que hay que dirigir, es un hombre"' (Cadavid Tobón, 1935: 198). Por ello, los deportes, la gimnasia, el canto, las excursiones, la gimnasia rítmica, la presentación de sencillos juguetes, la natación y todo este conjunto de vida sana y alegre componían las actividades que se realizaban en la clase de Educación Física.

Los ejercicios practicados en clase tenían como fin el desarrollo físico del niño, combinando los dos aspectos esenciales de la nueva escuela, el juego y el trabajo:

los ejercicios consistían en que los niños procuraran imitar los movimientos, saltos y carreras de algunos animales -conejo, rana, gato, ganso, perro, etc.- y también en lo que se refiere a los movimientos que ejecuta el hombre en el trabajo -cortar árboles, aserrar, martillar, apisonar, etc.- (Jaime, 1935: 603).

Las excursiones eran otra actividad de buen resultado, porque se hacían a manera de paseos, como complemento y aplicación de los centros de interés y del trabajo manual, iniciando a los niños en el conocimiento material de la región, de los elementos de vida práctica con que se contaba, despertando el amor por la naturaleza y el entusiasmo por el adelanto industrial y artístico que se constituían en fuentes de prosperidad y de riqueza (Jaime, 1935: 200).

Entre tanto, la práctica del escultismo constituyó un retorno a la vida vivificante de la naturaleza como reacción contra los vicios que el refinamiento de la sociedad moderna vivía acrecentando en los centros urbanos. Sin embargo, ningún sistema de educación física era mejor que el concebido por lord Baden Powel en su institución de los Boy Scouts, porque este sistema no solamente comprendía todos los ejercicios de campo y excursionismo, sino que dentro de él era posible el ejercicio de todos los deportes. El escultismo era sin duda el más simpático y atractivo de todos los ensayos pedagógicos modernos para niños y jóvenes de más de doce años. Constituía una escuela de altas virtudes cívicas y de nobles cualidades morales. La práctica del escultismo formaba hombres vigorosos, caracteres bien templados y caballeros integrales (Bernal Jiménez, 1935: 454).

En definitiva,

sin cultura física necesariamente la educación quedaría minada por su base, aunque se desplieguen todos los esfuerzos posibles para conseguir el descanso mental, pues los niños crecerán débiles e incapaces para realizar en la vida un trabajo constante y eficaz (Cadavid Tobon, 1935: 196).

Así que la práctica de los deportes, los juegos, el escultismo, las excursiones y los paseos hacían que el niño aprendiera, jugara y se proyectara como adulto del mañana, fortaleciendo su cuerpo y su voluntad, ya que la práctica constante de alguna de estas actividades requería la formación en la disciplina, aspecto importante para forjar personas de bien.

La Educación Física, pensada como clase escolar integradora entre la mente y el cuerpo, empezó a descifrarse como saber pedagógico importante para la escuela, con actividades que, además del fortalecimiento corporal, trabajaban para formar el carácter y la voluntad de los estudiantes, rompiendo con los viejos prejuicios escolares, cuando los ejercicios gimnásticos obedecían a una sistematización basada en un desarrollo animal, clases consideradas únicamente como simple descanso, sin interés ni importancia alguna. La nueva escuela proporcionó un valor distinto a la clase de educación física, ya que ésta desvirtuaba el mito de la separación mente-cuerpo, se preocupaba por el buen funcionamiento biológico del cuerpo del niño en la escuela, por la vida sana y alegre, por formar individuos productivos, fuertes y vigorosos, en procura de la redención de la raza. 
Así pues, las prácticas corporales fueron asumidas por la escuela interviniendo el cuerpo de los estudiantes, determinando un sujeto saludable, vigoroso, de carácter fuerte, respetuoso, luchando con la idea de regenerar una raza considerada pobre, ignorante y enfer-

\section{Referencias}

Arcila, P. (1935, enero-febrero). Acción social de la escuela. En: Revista Educación. III (18 y 19).

Arizala, P. E. (1935, enero-febrero). Acción social de la escuela. En: Educación. III (18 y 19).

Balcázar, L. (1935, enero-febrero). Las modernas orientaciones educativas. Educación, III (18 y 19).

Benavides, M. (1933, octubre). Localización de las facultades intelectivas. Educación, I (4).

Bernal Jiménez, R. (1935, julio-agosto). La Facultad de ciencias de la educación. Educación, III (24 y 25). Bogotá.

(1935, enero-febrero). El papel del médico escolar. Educación. III (18 y 19). Bogotá. ción, III (24 y 25).

(1935, julio-agosto) ¿Qué son los boy-scouts? Educa-

Cadavid Restrepo, T. (1933, septiembre). El apoyo a los escolares pobres y la educación especifica de los "sobresalientes". Educación, I (2).

Cadavid Tobón, A. (1935, marzo-abril). Excursiones Escolares. Educación, III (20 y 21).

Camargo Uribe, S. (1935, septiembre-octubre). Educación y progreso. Educación, III (26 y 27). Bogotá.

Castellanos, V. (1933, septiembre). Autodominio por la Educación. Educación, I (2).

Discurso sobre la educación. (1935, enero-febrero). Educación, III (18 y 19).

Ebratt, L. (1933, octubre). La reforma escolar ante los problemas nacionales emergentes. En: Ministerio de Educación. Programas para las escuelas primarias. Educación, I (5). ma; para ello se asumieron los preceptos de la escuela nueva, prácticas, conceptos y saberes innovadores que brindarían a la nación colombiana el progreso racial, económico y social que estaba buscando.

Gaviria, T. (1935, marzo-abril). La educación en el Huila. Educación, III ( 20 y 21$)$.

Gómez Vega, P. (1935, noviembre-diciembre). La Educación femenina en Colombia. Educación, III ( 28 y 29).

N. D. S. F. Circular del señor Director de Educación Pública del Departamento a las Juntas Escolares.

Nieto Caballero, A. (1933, octubre). Los centro de interés en la enseñanza primaria. Educación, I (5).

Nieto Caballero, A. (1935, julio-agosto). La educación nacional. Educación, III (24 y 25).

Pantoja, J. E. (1935, mayo-junio) Pedagogía Decroliana. Educación, III (22 y 23).

Reunión de directores de educación. Ministerio de Educación Nacional. (1935, julio-agosto). Educación, III (28 y 29).

Rojas, E. (1933, octubre). La preparación técnica del maestro. Educación, I (3).

Saldarriaga, O. (1997). El oficio de maestro. Bogotá: Cooperativa Editorial Magisterio.

Saldarriaga, O.; Sáenz, J.; y Ospina, A. (1997). Mirar la infancia: pedagogía, moral y modernidad en Colombia, 1903-1946. Vol. 2. Bogotá: Ediciones Foro Nacional por Colombia.

Velásquez, P. (1933, octubre). La dignificación del magisterio. Educación, I (5).

Zapata, L. (1935, enero-febrero). ¿Cómo se hacen los exámenes en la Maison Des Petites? Educación, III (18 y 19). 